\title{
Acute calcific tendonitis of dorsal interosseous muscles of the hand: uncommon site of a frequent disease
}

\author{
D. Schneider, M. Hirsch
}

Clínica Alemana de Temuco, Facultad de Medicina, Universidad del Desarrollo, Temuco, Chile

\begin{abstract}
SUMMARY
Acute calcific tendinopathy is one of the manifestations of hydroxyapatite crystal deposition disease. While it is more frequent in the shoulder, it has been described in virtually all areas of the body, but rarely in the muscles of the hand. Its etiopathogenesis is not yet fully understood and despite being a fairly frequent condition, it is commonly misdiagnosed. The onset of the disease is usually acute and resolves spontaneously. Acute calcific tendinitis of the interosseous tendons of the hand is an uncommon site of a frequent condition. The clinical presentation is similar to other entities, thus errors in diagnosis frequently occur, resulting in over-treatment or unnecessary tests.

We describe a case of acute calcific tendinitis of the interosseous muscles of the hand with a brief review of the current literature with emphasis on diagnostic imaging methods.
\end{abstract}

Key words: Calcific Tendonitis; Radiography; Ultrasound; Hydroxyapatite crystal; Deposition disease.

Reumatismo, 2017; 69 (1): 43-46

\section{INTRODUCTION}

cute calcific tendonitis of the interosseous tendons of the hand is an uncommon site of a frequent condition (1). Symptoms develop acutely and typically resolve spontaneously or with conservative management over the course of 3 to 4 weeks. Cases of slower evolution with a tendency to chronicity have been described (2).

\section{CASE REPORT}

A 68-year-old female patient with chronic hypertension, type II diabetes mellitus, chronic heart failure, and bronchial asthma under treatment and compensated, appeared with a 3-week history characterized by sudden onset pain, increased volume and functional impotence of the $2^{\text {nd }}$ and $3^{\text {rd }}$ right metacarpophalangeal joints.

An ultrasound (US) of the hand was obtained, which demonstrated juxta-articular calcifications in the lateral margin of the metacarpophalangeal joints of the index and middle fingers, in relation to the ten- dons of the dorsal interosseous muscles, without signs of metacarpophalangeal synovitis (Figure 1). Extensor and flexor tendons showed no ultrasound abnormalities. There was also mild synovial thickening of the radiocarpal joint. The increased volume of the joint referred by the patient was not clearly visible at time of the US, probably due to the beginning of symptomatic treatment prior to the examination.

The study was then complemented with anteroposterior and oblique hand radiographs, which showed periarticular calcifications in the same locations described in the US (Figure 2), and some discrete degenerative compromise of the trapeziometacarpal joint and some distal interphalangeal joints.

The patient was treated conservatively with nonsteroidal anti-inflammatory drugs (NSAIDs) and physical therapy, evolving with partial resolution of symptoms. Two months after beginning the treatment, she showed no pain in her middle finger, with only a partial decrease of the symptoms on the index finger.
Corresponding author Daniel Schneider

Clínica Alemana de Temuco, Facultad de Medicina, Universidad del Desarrollo, Senador Estebanez 645 , Temuco, Chile, 4810297 E-mail: danschneider@alemana.cl 

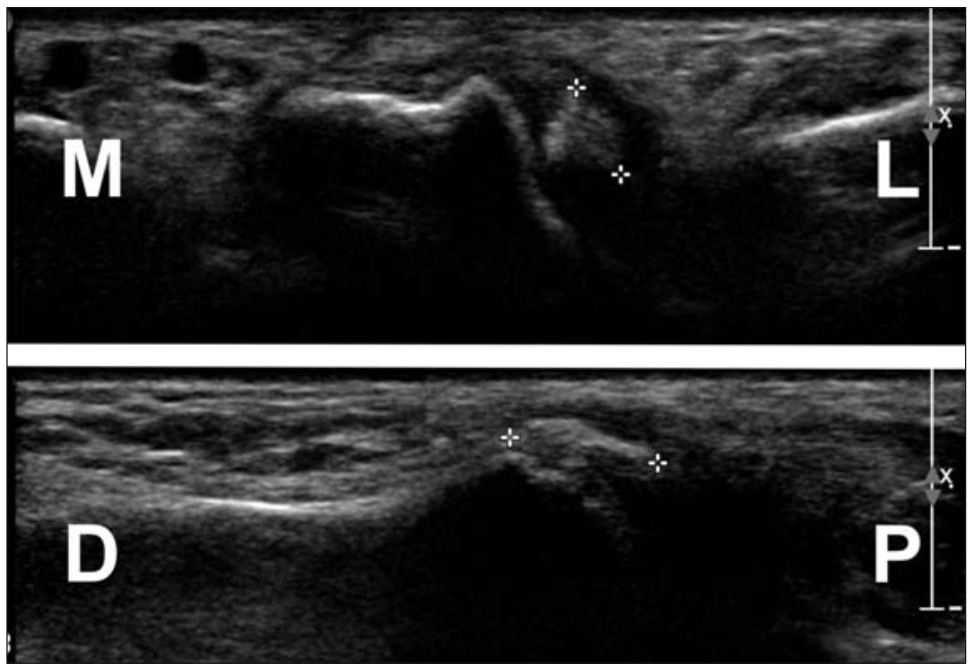

Figure 1 - Right hand US. Top: Transverse plane by a dorsal approach shows calcification in the tendon of the second dorsal interosseous muscle, lateral to the base of the proximal phalanx. Bottom: Coronal plane by a lateral approach at the metacarpophalangeal joint of the index finger, shows calcification in the tendon of the first dorsal interosseous muscle (M: medial, L: lateral, D: distal, P: proximal).

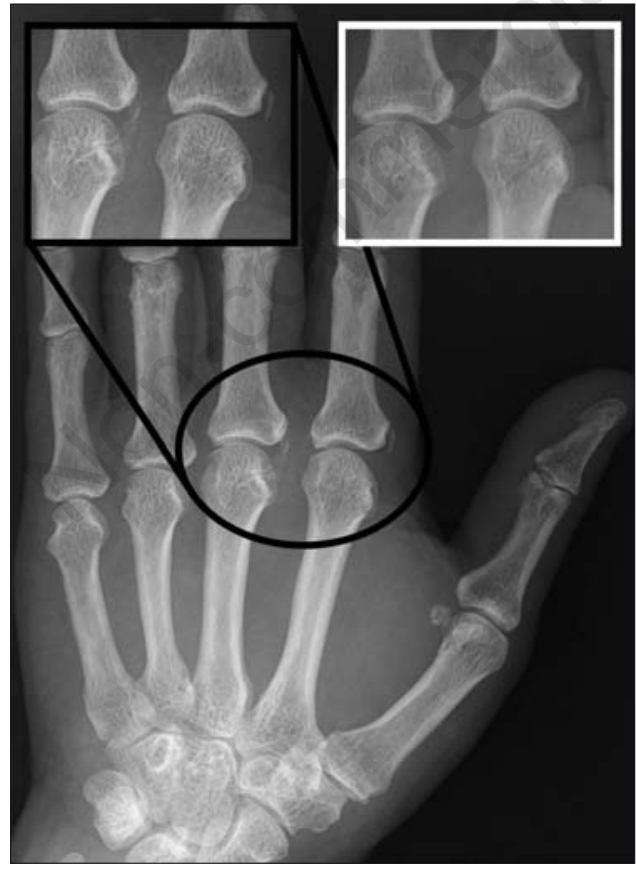

Figure 2 - Right hand anteroposterior radiograph with zoom in the region of interest. Calcific tendinopathy of the first and second dorsal interosseous tendons at first control (black box). Follow-up radiograph at 4 months, with partial reabsorption of the second dorsal interosseous muscle calcifications (white box).
Four months from the onset of symptoms the patient persisted with pain in the index finger. Follow up radiographs were performed, which showed a decrease in calcification size in relation to the base of the proximal phalanx of the middle finger, without significant changes in calcification in relation to the base of the proximal phalanx of the index finger (Figure 2).

\section{DISCUSSION AND CONCLUSIONS}

Calcific tendinitis is a common, but poorly understood and frequently misdiagnosed condition (3), characterized by the deposition of hydroxyapatite crystals in tendon insertions. It has been referred to in multiple terms including: calcific periarthritis, calcareous tendinitis, calcareous periarthritis and rheumatism by hydroxyapatite. Currently, acute calcific tendinitis is considered a subset of hydroxyapatite deposition disease, in addition to other manifestations of this entity, such as periarticular deposits and hydroxyapatite-induced arthritis (3-5). The pathogenesis of the disease is not fully understood but associations with alterations in vascularization, hypoxia, trauma, and metabolic disorders have been postulated $(2,5)$. Recent evidence suggests that stressors trigger metaplasia with deposition and reabsorption of calcium, mediated by cells $(2,6)$.

Although it more frequently affects the shoulder, cases have been described in almost every area of the body, with a prevalence of $2 \%$ in the hand and wrist (5-7). The most frequent locations in the hand and wrist are the flexor carpi ulnaris tendon, extensor carpi ulnaris and the flexors and extensors of the fingers. They have also been described in the interphalangeal, metacarpophalangeal and dorsal wrist joints $(3,6,8,9)$, very rarely in the carpal tunnel (10), long extensor of the thumb (11), and tendons of the intrinsic muscles (12). Furthermore, it can occur simultaneously in several locations $(5,13)$.

Acute calcific tendinitis is most commonly described in women and has a female-male ratio ranging from $2: 1$ to $5: 1(1,3,6)$. The 
mean age of onset is 45 years $(3,6,8)$, although there are also exceptional cases described in children $(6,11)$.

The differential diagnoses include pathologies such as calcium pyrophosphate dihydrate deposition disease, gout, infections, fractures, metabolic disorders and collagen vascular diseases (14). Therefore, diagnostic errors often result in overtreatment or unnecessary tests $(8,10)$. In most cases, well-localized symptoms and signs of rapid onset, including pain, local heat, functional limitation, soft tissue swelling and erythema are seen. Typically, a gradual reduction of symptoms is seen from the first 4-7 days with full resolution within the first 3-4 weeks. Treatment includes NSAIDs, modification of activities or use of splints during the acute period. Moreover, infiltrations of anesthetics with or without corticosteroids have also been described, and have shown a good symptomatic response (3, 6). Some authors have reported persistence of symptoms for several months, with discrepancies as to whether it is a different entity or whether some associated conditions may delay the resolution (2). Some patients show recurrence at different locations (1). The standard of reference for the diagnosis is represented by demonstration of crystals in the joint synovial fluid by polarizing light microscopy (15), but it is not always performed due to its technical difficulty or because the absence of effusion.

Microbiological and basic laboratory tests are usually normal (8).

A simple radiography can detect calcifications and allow an appropriate diagnosis. Calcifications are almost always visible and generally it is the only test required (7). The findings include dense, homogeneous and amorphous calcium deposits, usually round or oval, without cortex or internal trabecula, periarticular or in relation to tendons and ligaments, measuring between 2 to $10 \mathrm{~mm}$ with a morphology that can change over time (3). This characteristic homogeneous cloudlike appearance in the characteristic sites without an underlying disorder distinguishes hydroxyapatite deposition disease from most other disorders (14).
US is useful for diagnosis, tendon evaluation and guidance of therapeutic procedures, especially in the shoulder. The findings correspond to hyperechogenic calcification foci with or without acoustic shadow, tendon thickening and increased color Doppler vascularization $(7,13,15)$. The use of US to detect calcium deposits in asymptomatic patients has been described, but its usefulness has not been well-established (6).

Computed tomography (CT) is superior to conventional radiography for detecting and delineating intratendinous calcifications and it is recommended in cases where the radiography failed to detect them (7). Calcium tendonitis may occasionally have an aggressive appearance in the images, showing extensive soft tissue alterations or bone changes such as erosion and periosteal reaction. In these cases, CT is the best modality to evaluate this involvement. This aggressive appearance should not be confused with infections or malignant diseases. Tendon localization, absence of joint effusion, or soft tissue masses may help to make the correct diagnosis (13).

Findings using magnetic resonance imaging (MRI) have been sporadically reported. Calcifications are seen as hyper-intense areas in all imaging sequences. Other findings include edema or soft tissue inflammatory changes and bone edema, which may falsely direct diagnosis to infection or other diseases $(3,7)$.

Acute calcific tendinitis has a clinical course and characteristic radiological findings. A detailed history together with diagnostic images, in most cases, allows an adequate diagnosis, avoiding unnecessary tests and treatments.

Conflict of interest: the authors declare no potential conflict of interest.

\section{REFERENCES}

1. Arandas FDS, Santos FADS, de Souza SP, et al. Acute calcific periarthritis of the hand. J Clin Rheumatol. 2005; 11: 223-4.

2. Harvie P, Pollard TCB, Carr AJ. Calcific tendinitis: natural history and association with endocrine disorders. J Shoulder Elb Surg. 2007; 16: 169-73. 
3. Doumas C, Vazirani RM, Clifford PD, Owens P. Acute calcific periarthritis of the hand and wrist: a series and review of the literature. Emerg Radiol. 2007; 14: 199-203.

4. Bonavita JA, Schumacher HR. Hidroxyapatite deposition disease. Radiology. 1980; 134: 621-5.

5. Hayes CW, Conway WF. Calcium hydroxyapatite deposition disease. Radio Graphics. 1990; 10: 1031-48.

6. Nikci V, Doumas C. Calcium deposits in the hand and wrist. J Am Acad Orthop Surg. 2015; 23: 87-94.

7. Lee HO, Lee YH, Mun SH, et al. Calcific tendinitis of the hand and foot : a report of four cases. J Korean Soc Magn Reson Med. 2012; 16: 177-83.

8. Moradi A, Kachooei AR, Mudgal CS. Acute calcium deposits in the hand and wrist. J Hand Surg Am. 2014; 39: 1854-7.

9. Ali SN, Kelly JL. Acute calcific tendinitis of the finger-a case report. Hand Surg. 2004; 9: 105-7.

10. Harris AR, McNamara TR, Brault JS, Rizzo M. An unusual presentation of acute calcific tendinitis in the hand. Hand. 2009; 4: 81-3.

11. Hakozaki M, Iwabuchi M, Konno S, Kikuchi $\mathrm{S}$. Acute calcific tendinitis of the thumb in a child: a case report. Clin Rheumatol. 2007; 26: 841-4.

12. Greene TL, Louis DS. Calcifying tendinitis in the hand. Ann Emerg Med. 1980; 9: 438-40.

13. Siegal DS, Wu JS, Newman JS, et al. Calcific tendinitis: a pictorial review. Can Assoc Radiol J. 2009; 60: 263-72.

14. Steinbach, LS. Calcium pyrophosphate dihydrate and calcium hydroxyapatite crystal deposition diseases: imaging perspectives. Radiol Clin N Am. 2004; 42: 185-205.

15. Paparo F, Fabbro E, Ferrero G, et al. Imaging studies of crystalline arthritides. Reumatismo. 2011; 63: 263-75. 